\title{
REFERENCE CONVOLUTION OF LOW RESOLUTION FIDS IN THE PRESENCE OF POOR MAGNETIC FIELD HOMOGENEITY
}

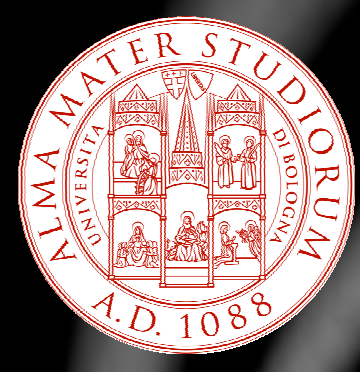

\author{
M. A. Cremoninia, L. Venturia, S. Sýkorab, \\ C. Cavania, \\ G. Placuccia \\ ${ }^{a}$ Department of Food Science, University of Bologna, Italy ${ }^{b}$ Extra Byte, Castano Primo, Italy
}

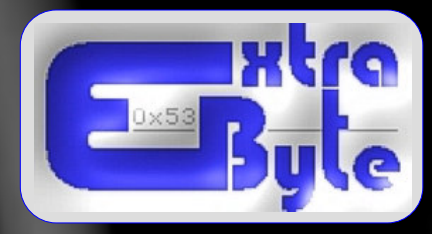

\section{INTRODUCTION}

Quantification of proton FID signals in time-domain NMR is mostly done by fitting the experimental data to a discrete number of suitable decay functions while ignoring any frequency differences between their individual components. The latter simplification is justified when measuring systems with inherently broad lines (solids, gels, tissues, etc.) using lowfield $(<0.5 \mathrm{~T})$ time-domain instruments. Instruments of this type, however, are typically built around permanent magnets with no shimming facilities. As a result, the measured FID's of all samples - but the most rigid solids - are heavily and unpredictably distorted by magnetic field inhomogeneity and approximating them by meaningful fitting functions is often nearly impossible.

\section{AlM OF THE WORK}

We show that a technique known in high resolution NMR as reference deconvolution [1] can be modified and used to fit distorted FIDs to any desired theoretical model. The essence of the proposed reference convolution method (see the Appendix for details) is to convolute the theoretical model data with an intense experimental signal (for example a water signal) prior to comparing them with an actual FID.

\section{EXPERIMENTAE}

The new protocol was tested using the FIDs of two samples, of bovine serum albumin (BSA) gels obtained, respectively, from two equiconcentrated $\mathrm{H}_{2} \mathrm{O}$ (sample $\mathrm{A}$ ) and $\mathrm{D}_{2} \mathrm{O}$ (sample B) BSA solutions with $\mathbf{p H}=\mathbf{8}$ by heating them at $75^{\circ} \mathrm{C}$ for 12 minutes. In the Figure, the two FID's are shown as black lines.

\section{THEORETICAL}

The theoretical model consisted in both cases of one 'fast' and one 'slow' Lorentzian line. During the fitting process, given the model parameters (component intensities and linewidths), one generates the respective theoretical decay data and convolves them with an experimental water signal according to Equation (3) prior to each square-deviation comparison with the experimental FID's.

\section{RESULTS AND CONCLUSIONS}

The ratio between the intensity of the 'fast' and the 'slow' components in sample A matched the ratio between the BSA and water protons (23.7 to 24.1$)$, indicating that the exchange between solvent water and exchangeable BSA protons is quite slow, in agreement with previous studies [2-4]. Further, on passing from $\mathrm{H}_{2} \mathrm{O}$ to $D_{2} O$ the 'fast' signal decreases by about $17.8 \%$, an amount comparable to the percentage of exchangeable protons in the BSA $(19.3 \%)$ [2]. These results illustrate the usefulness of the reference convolution method for the quantification of proton pools in highly distorted FID's.

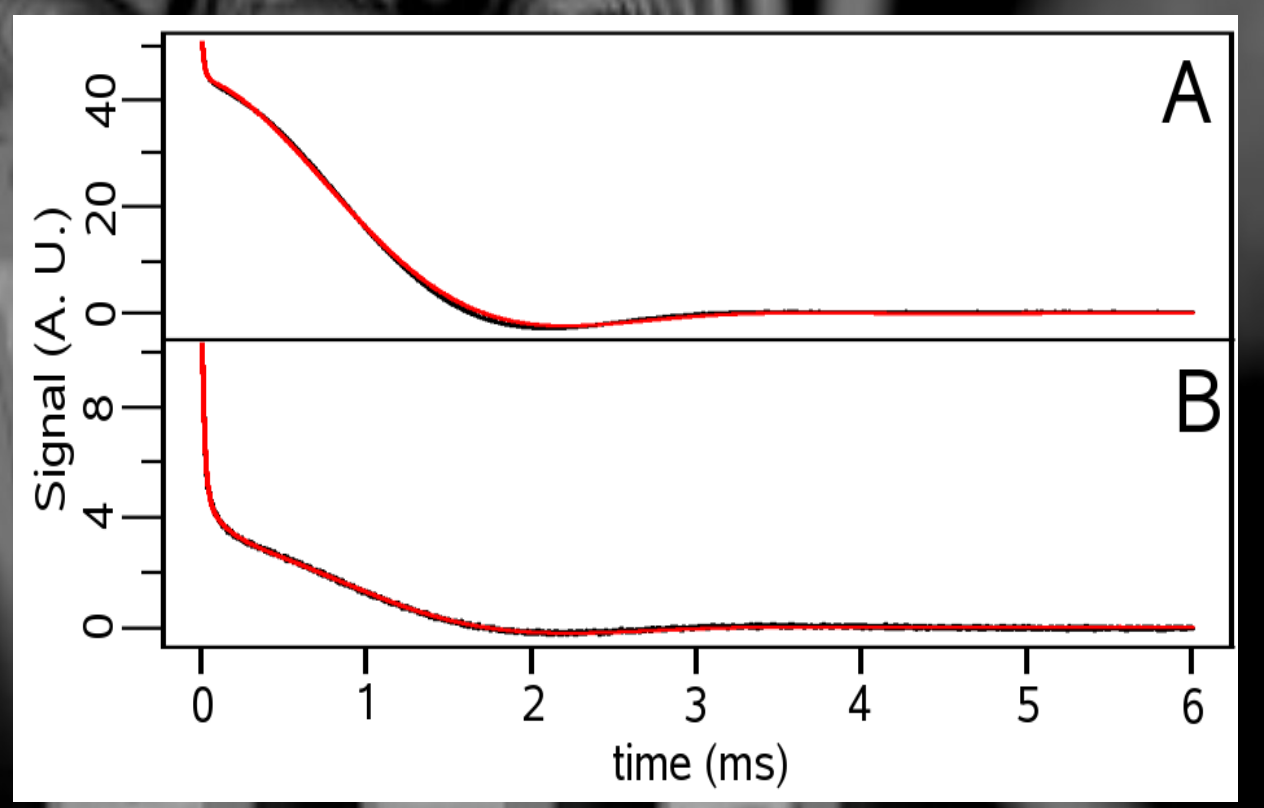

FID's (black lines) of BSA gel samples prepared in protonated (A) and deuterated (B) water buffer solution. The red lines are the fitted curves corresponding to a simple theoretical model and convoluted with an experimental water signa.

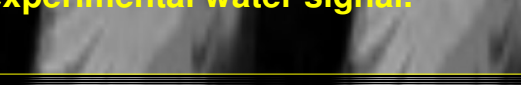

\section{APPENDIX}

The reference convolution technique [5] starts from the hypothesis that the effect of the magnetic field inhomogeneity on the FED is independent of the sample; an experimental FID $\left(F_{1 D_{e x p}}\right)$ is thus the point by point multiplication of the undistorted FID (FID und by an unknown distortion function D:

$$
\mathrm{FID}_{\text {exp }}=\mathrm{FID}_{\text {und }} \cdot \mathrm{D}
$$

To get rid of D, the FID of a water sample with the same geometry of the previous sample is recorded in the same experimental conditions. The experimental water FID $\left(\right.$ FID $\left._{w}\right)$ can be expressed as:

$$
\mathrm{FID}_{\mathrm{w}}=\mathrm{I}_{0 \mathrm{w}} \mathrm{e}^{-\mathrm{R}_{2 \mathrm{w}} \mathrm{t}} \cdot \mathrm{D}
$$

where $\mathbf{R}_{2 w}$ is the water transverse relaxation rate. If for example all components $(i, i=1,2, \ldots)$ of the $F_{1} D_{\text {und }}$ are exponential, Equation $(1)$ can be rewritten as:

$$
\mathrm{FID}_{\exp }=\sum_{\mathrm{i}} \frac{\mathrm{I}_{0 \mathrm{i}}}{\mathrm{I}_{0 \mathrm{w}}} \mathrm{e}^{-\left(\mathrm{R}_{2 \mathrm{i}}-\mathrm{R}_{2 \mathrm{w}}\right) \mathrm{t}} \cdot \mathrm{FID}_{\mathrm{w}} \text { (3) }
$$

In frequency domain, this would be equivalent to the convolution between an undistorted spectrum and the spectral line of water acting as a distortion reference (whence the name). 